\title{
Adaptation des formes de financement agricole au changement climatique : cas du warrantage au Bénin
}

Janvier Egah

Janvier Egah : PhD. Enseignant chercheur à l’Université de Parakou, Bénin Laboratoire SociétéEnvironnement (LaSEn) Nationalité : Béninoise. Adresse professionnelle : Faculté d'Agronomie, Université de Parakou, Parakou, Bénin. Email : egahjanvier@gmail.com; janvier.egah@fa-up.bj

Tél : +229 96371779

DOI: $10.25518 / 2295-8010.1923$

\section{Résumé :}

Les défis du changement climatique nécessitent des formes de financement agricole adaptées. L'étude a analysé l'adaptabilité des formes de warrantage au changement climatique au Bénin. Les données sur les promoteurs du warrantage, sur les parties prenantes et leurs rôles, sur les difficultés et risques liés au warrantage ainsi que sur leurs relations avec les changements climatiques ont été collectées auprès des acteurs à l'aide d'un guide d'entretien. L'analyse des discours et des contenus a permis de relever les facteurs d'inadéquation du warrantage face au changement climatique. Les acteurs impliqués dans les formes de warrantage sont les institutions financières, les organisations des producteurs et les services d'encadrement de l'État jouant respectivement le rôle de mise en place du crédit, d'établissement du cautionnement solidaire, de la mise en marché et l'appui-conseil en conditionnement des produits warrantés. Les risques climatiques et du marché ne sont pas couverts. Les formes actuelles de warrantage ne sont pas adéquates face au changement climatique.

Ainsi, l'adaptation des formes de warrantage au changement climatique nécessite l'implication d'une compagnie d'assurance pour assurer les risques climatiques et d'un service météorologique qui fournira les informations climatiques aux producteurs. Les services de vulgarisation fourniront des appuis-conseils complémentaires sur l'accès aux variétés tolérant la sécheresse.

Mots-clés : adaptation, financement agricole, warrantage, changement climatique, risques

\section{Abstract :}

Adaptation of agricultural finance to climate change: the case of warrantage in Benin.

The challenges of climate change require adapted forms of agricultural finance. The study analyzed the adaptability of forms of warrantage to climate change in Benin. Data on promoters of the warrantage, stakeholders and their roles, difficulties and risks linked to the warrantage and their relationship with climate change were collected from direct stakeholders using an interview guide. The analysis of discourses and contents made it possible to identify the factors that make the current forms of warrantage unsuitable to face climate change. Actors involved in warrantage are financial institutions, producer organizations and government support services respectively playing the role of setting up credit facilities, establishing joint guarantee, marketing and providing advice on the packaging of warranted products. Climate and market risks are not covered. The current forms of warrantage are not adequate to face climate change. 
Adaptation des formes de financement agricole au changement climatique : cas ...

Thus, adapting the forms of warrantage to the climate change requires the involvement of an insurance company to insure the climatic risks and a meteorological service to provide climatic information to producers. Extension services will provide additional advisory support on access to drought tolerant varieties.

Keywords : adaptation, agricultural finance, warrantage, climate change, risks

\section{Introduction}

Les enjeux majeurs économiques et sociaux du changement climatique nécessitent des adaptations afin de réduire non seulement l'émission des gaz à effet de serre mais aussi les impacts sur la population (1). Dans ce contexte du changement climatique, l'accès au financement et la mise en marché des produits agricoles sont des contraintes qui freinent l'adaptation des agriculteurs et agricultrices dans la plupart des pays de l'Afrique subsaharienne qui sont fortement affectés par les affres des changements climatiques $(2 ; 3)$. Comme mentionné dans un rapport de la FAO, les investissements agricoles jouent un rôle essentiel dans la promotion de la croissance agricole, dans la réduction de la pauvreté et de la faim (4).

Le même rapport indique que ces investissements sont aux mains des producteurs agricoles dont les capacités sont limitées surtout dans un contexte de changement climatique. À ce titre, le développement des innovations agricoles doit intégrer les aléas du changement climatique afin de réduire la vulnérabilité des agriculteurs et agricultrices $(1 ; 5)$. Ainsi, la question de l'adaptation des innovations relatives aux formes de financement agricole émerge. Pour analyser cette question, cette étude part des formes de warrantage mises en œuvre au Bénin pour analyser leur adéquation avec les réalités du changement climatique.

Au Bénin, l'agriculture contribue au PIB national à concurrence de près de $27 \%$ (6). La Stratégie Nationale du Conseil Agricole (SNCA) fait état de ce que l'agriculture joue un rôle économique important au Bénin par le fait qu'elle emploie environ $70 \%$ de la population active et fournit environ $75 \%$ des recettes d'exportation et $15 \%$ des recettes de l'Etat (7). Aussi, contribue-t-elle significativement à la lutte contre la pauvreté et l'insécurité alimentaire (7). Cependant, l'analyse diagnostique du secteur agricole a révélé que l'insuffisance et l'inadéquation du financement du secteur agricole, l'absence de stratégie nationale de financement du secteur agricole et les difficultés d'accès au marché sont entre autres les maux qui minent l'agriculture (8). De plus, la politique de crédit formel de trésorerie est absente ou inadaptée aux conditions des paysans agricoles (9). La difficulté d'accès au financement par les producteurs agricoles s'explique surtout par la réticence des institutions financières à investir dans le crédit rural (10). Cette réticence est due à l'accroissement des coûts opérationnels et du risque liés à l'agriculture et spécifiquement au changement climatique. La plupart des producteurs agricoles font recours à des crédits informels avec un taux d'intérêt annuel pouvant dépasser $200 \%$; ce qui les maintient dans une trappe d'endettement et de pauvreté (9).

Les conditions actuelles offertes par les institutions financières aux producteurs agricoles ne garantissent pas un financement durable, le développement de l'agriculture et l'amélioration du bien-être des populations rurales (2). De plus, le manque d'organisation des producteurs sur le marché ne permet pas à ces derniers de profiter des meilleurs prix (11). En corollaire, la capacité des agriculteurs est donc limitée pour faire face aux effets néfastes du changement climatique (11). 
De ce fait, il faut trouver un mécanisme qui répond non seulement aux besoins de financement des agriculteurs mais aussi au problème de mise en marché des produits agricoles dans un contexte de changement climatique. Cette préoccupation est d'autant plus importante que le Ministère de l'Agriculture, de l'Élevage et de la Pêche l'a inscrit dans le Plan Stratégique du Développement du Secteur Agricole (PSDSA) à l'horizon 2025. Ainsi, le système de warrantage apparaît comme une innovation pour sortir les producteurs des cercles vicieux liés au financement et à la mise en marché

Le warrantage est un système dans lequel les producteurs accèdent au crédit contre une garantie constituée de stock de produits vivriers (12). Le warrantage permet aux producteurs de bénéficier de meilleurs prix et d'éviter de brader les productions agricoles juste après les récoltes. Il constitue également une opportunité d'écoulement des produits agricoles à travers la mise en marché collective des produits warrantés (12). La plupart des études menées sur le système de warrantage ont analysé la performance et les déterminants d'adoption des formes de warrantage comme une innovation institutionnelle $(12 ; 13 ; 14)$. Mais, la prise en compte du changement climatique dans l'analyse des formes de warrantage a été occultée de ces études. De plus, plusieurs études scientifiques ont été faites sur les stratégies d'adaptation des agriculteurs au changement climatique sans intégrer les questions de financement (3).

Toutefois, la question du financement des adaptations au niveau des ménages, tout aussi importante que les stratégies, n'a été que très peu abordée dans la recherche. Bien que le système de warrantage procure des avantages aux producteurs et que les questions de financement sont importantes pour faire face au changement climatique, il convient de se demander si les formes de warrantage répondent efficacement aux besoins des producteurs dans un contexte de changement climatique au Bénin $(3 ; 15)$. Le financement de l'adaptation au changement climatique est l'une des conditions pour réduire le niveau de vulnérabilité des producteurs (16). Les questions abordées dans cet article sont les suivantes: Quels sont les acteurs impliqués dans la mise en ouvre du warrantage et leurs rôles? Les questions relatives aux changements climatiques sont-elles prises en compte dans le système de warrantage ? La présente étude vise en outre à analyser l'adéquation des formes de financement agricole vis-à-vis du changement climatique en partant des formes de warrantage actuelles et à proposer des adaptations possibles pour améliorer leur efficacité.

\section{Matériel et méthodes}

\section{Choix et présentation de la zone d'étude}

Létude a été réalisée au Bénin dans les communes où le warrantage est mis en œuvre. Il s'agit des communes de Zogbodomey, de Sinendé et de Kalalé. Ces communes ont été choisies parce qu'elles ont été les pionnières dans la mise en œuvre du warrantage au Bénin. L'Union Communale des Producteurs de Zogbodomey (UCP-Z), l'International Fertilizer Development Center (IFDC) et le Projet d'Appui à la Décentralisation, à la Déconcentration et au Développement Économique Local (PA3D) ont démarré le warrantage respectivement à Zogbodomey en 2010, à Sinendé en 2009 et à Kalalé en 2008. Ces communes d'étude appartiennent à des régions différentes mais présentent des similitudes agricoles comme suit :

- La commune de Zogbodomey, située dans le département du Zou à $150 \mathrm{~km}$ environ de Cotonou, est comprise entre $6^{\circ} 56^{\prime}$ et $7^{\circ} 08$ de latitude Nord, $1^{\circ} 58^{\prime}$ et $2^{\circ} 24^{\prime}$ de longitude 
Est. Elle est exposée à un climat de type subéquatorial avec une alternance de deux saisons pluvieuses et deux saisons sèches. Les terres cultivables occupent $60 \%$ de la superficie totale qui s'élève à $825 \mathrm{~km}^{2}$. La production végétale occupe près de $80 \%$ de la population agricole avec des techniques culturales archaïques. Les principales cultures sont : le maïs, le niébé, l'arachide, le manioc et le coton. On cultive également le soja, le riz et les produits maraîchers.

- Localisée au nord-ouest du département du Borgou, la commune de Sinendé s'étend sur une superficie de $2.289 \mathrm{~km}^{2}$, soit 8,85 \% de la superficie du département. Elle bénéficie d'un climat de type soudano-guinéen avec une saison pluvieuse s'étendant d'avril à octobre et d'une saison sèche allant de la mi-octobre à la mi-avril.

- Située au nord-est du département du Borgou, la commune de Kalalé s'étend sur une superficie de $3586 \mathrm{~km}^{2}$ représentant 13,87 \% de la superficie du département. Le climat est de type soudano -sahélien caractérisé par une saison pluvieuse d'avril à octobre et une saison sèche de novembre à mars.

Dans chacune des trois communes, se trouve une structure déconcentrée du Ministère de l'Agriculture, de l'Élevage et de la Pêche (MAEP) appelée Secteur Communal de Développement Agricole (SCDA) et des institutions de microfinance. Les institutions de microfinance sont les Caisses Locales de Crédit Agricole Mutuel (CLCAM) dans les communes de Sinendé et de Zogbodomey et l’ONG SIA N’SON Microfinance dans la commune de Kalalé.

Par ailleurs, deux villages par commune ont été choisis en fonction du nombre de producteurs et productrices ayant participé au warrantage. Autrement dit, les villages retenus ont été ceux dans lesquels le nombre de déposants est le plus élevé dans la commune. Il s'agit des villages de Basso (59 déposants) et de Bouca (24 déposants) dans la commune de Kalalé, de Sèkèrè (30 déposants) et de Yara (98 déposants) dans la commune de Sinendé et enfin de Agoïta (16 déposants) et de Dèmè (15 déposants) dans la commune de Zogbodomey.

\section{Unités de recherche et échantillonnage}

Les unités de recherche ont été les divers types d'acteurs impliqués dans chaque forme de warrantage. Il s'agit des promoteurs (IFDC, UCP-Z, PA3D), des Secteurs Communaux pour le Développement Agricole (SCDA) représentés par des agents de conditionnements à Sinendé et à Kalalé et par le Technicien Spécialisé en Production Végétale (TSPV) à Zogbodomey, des membres des comités de gestion (président, secrétaire) et des responsables des institutions de microfinance (IMF). Les CLCAM ont été les IMF impliquées dans les formes de warrantage promues par le Projet Intrants Non Coton (PINC)/ IFDC et par l'UCP- Z alors que SIA N'SON ONG a été l'IMF impliquée dans la forme de warrantage de PA3D. L'échantillonnage non probabiliste a été utilisé pour choisir ces acteurs de façon raisonnée en tenant compte de leur implication réelle dans la mise en œuvre du warrantage. Tous les acteurs institutionnels qui appuient l'opération dans chaque commune ont été enquêtés. Les deux responsables d'OP impliqués dans le comité de gestion ont été enquêtés. Quant aux déposants, la méthode de saturation des informations a été utilisée pour enquêter 15 déposants par village. Au total, 60 acteurs ont été enquêtés à raison de 20 acteurs (15 déposants et 5 acteurs institutionnels) par commune (Tableau 1). 
Tableau 1 : Taille de l'échantillon

\begin{tabular}{|c|c|c|c|c|}
\hline Types d'acteurs rencontrés & Zogbodomey & Sinendé & Kalalé & Total \\
\hline Promoteurs & 1 & 1 & 1 & 3 \\
\hline Institutions de microfinance & 1 & 1 & 1 & 3 \\
\hline Structures étatiques & 1 & 1 & 1 & 3 \\
\hline Comités de gestion & 2 & 2 & 2 & 6 \\
\hline Déposants/ producteurs & 15 & 15 & 15 & 45 \\
\hline Total & & & & \\
\hline
\end{tabular}

\section{Collecte et analyse de données}

Les informations collectées concernent les mécanismes de mise en œuvre des formes de warrantage, les types d'acteurs impliqués et leurs rôles, les difficultés et les risques rencontrés dans la mise en œuvre du warrantage et leurs relations avec le changement climatique, les manifestations du changement climatique et les possibilités d'adaptation du warrantage au changement climatique. Elles ont été collectées auprès des acteurs directs lors des entretiens de groupe à l'aide d'un guide d'entretien.

Ces données collectées ont fait l'objet d'une analyse des discours et des contenus. L'analyse causale a été élaborée avec les acteurs pour ressortir les causes des difficultés ou risques encourus par les producteurs et leurs relations avec les manifestations du changement climatique dans les communes.

\section{Résultats}

\section{Mécanisme de mise en ouvre du warrantage au Bénin}

Dans les trois communes étudiées, trois formes de warrantage ont été observées : warrantage AGR (activités génératrices de revenu), warrantage intrants et warrantage commercialisation, respectivement mis en œuvre à Kalalé, Sinendé et Zogbodomey. La mise en œuvre du warrantage tourne principalement autour du maïs, souvent associé à d'autres spéculations comme le soja, l'arachide, le niébé, le piment, etc. Les produits agricoles sont essentiellement collectés de décembre à mi-février. Ils sont stockés dans un magasin après le contrôle du taux d'humidité et de la qualité des produits à stocker par un agent de conditionnement du Secteur Communal de Développement Agricole (SCDA). Le magasin est doublement fermé à l'aide de deux cadenas. Les clés d'un cadenas sont gardées par les responsables d'organisations des producteurs (OP) alors que les clés de l'autre sont gardées par l’IMF pour empêcher l'ouverture unilatérale des magasins. Le dépôt de tout produit est sanctionné par le certificat de dépôt délivré à chaque déposant. Le certificat de dépôt renseigne sur le produit stocké, la quantité et le prix du produit au moment de 
la collecte des produits. Après la fermeture du magasin, l'IMF accorde des crédits aux producteurs déposants à un taux d'intérêt donné contre le certificat de dépôt. La valeur du crédit représente environ 80 \% au plus de la valeur du produit au prix du marché au moment de la collecte.

Le crédit peut être un crédit intrant, crédit AGR (activité génératrice de revenu) ou crédit de commercialisation. Un comité composé généralement des responsables des OP, du chargé de prêt de l'IMF et de l'agent de conditionnement de l'État est mis en place pour suivre les produits stockés et l'évolution de leurs prix sur le marché. Le magasin est dénoué après l'avis concerté des OP et de l'IMF. Les produits stockés sont vendus de façon groupée à un commerçant qui paie à l'IMF sous le contrôle du comité. L'IMF récupère son capital avec intérêt et met le revenu complémentaire dans le compte de l'OP. Les responsables des OP font la redistribution en fonction de la quantité de produit déposé après avoir déduit les frais de fonctionnement, de stockage des produits et de manutention selon le cas. Le processus dure généralement 4 à 6 mois selon les opportunités du marché.

En définitive, le warrantage permet aux ménages ruraux de bénéficier de crédits (en nature sous forme d'engrais et en espèce) en mettant en garantie leurs produits agricoles (vivriers). Certes, la mise en œuvre du warrantage semble être standard mais elle présente des caractéristiques variées selon le promoteur, les types d'acteurs impliqués, les paramètres financiers, l'échelle géographique d'application et la durée de l'expérience. Le mécanisme de warrantage est construit autour de trois types d'acteurs (OP, SCDA, IMF) (Figure 1).

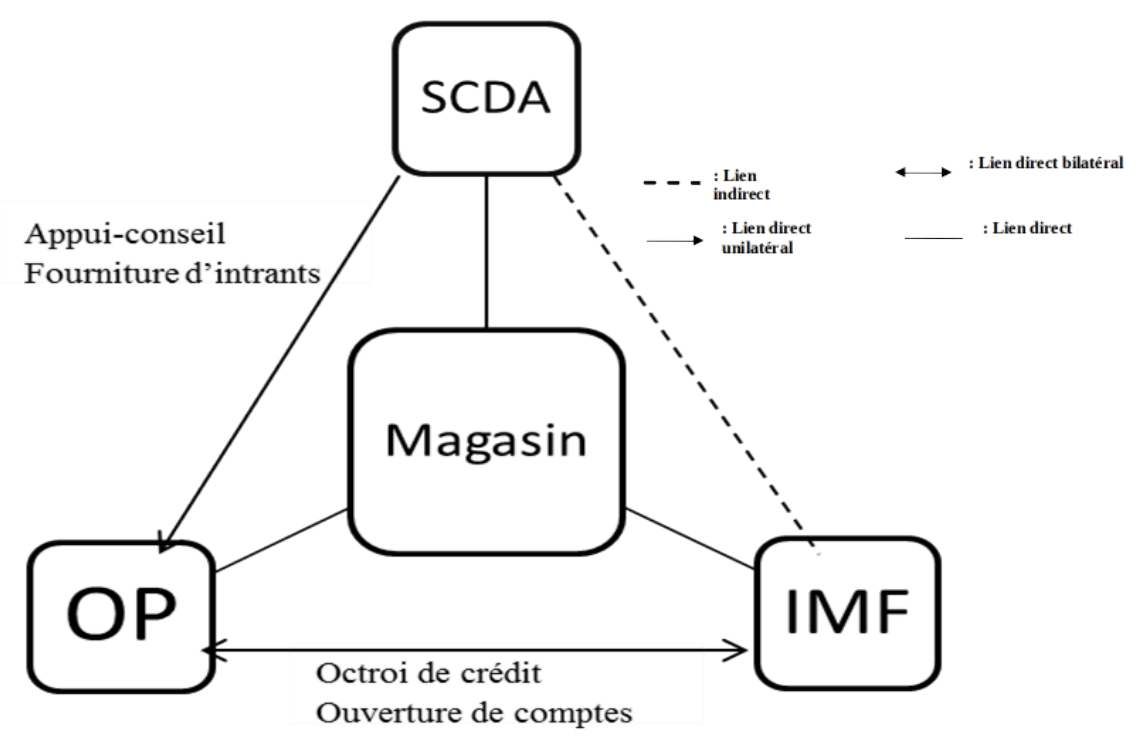

Figure 1 : Schématisation du modèle de warrantage dans les communes étudiées

\section{Acteurs et rôles dans la mise en ouvre du warrantage}

Dans les trois formes de warrantage analysées, les types d'acteurs ne sont pas les mêmes. En général, les types d'acteurs sont les promoteurs, les OP, les collectivités locales, les IMF et le service déconcentré de l'Etat (SCDA) (Tableau 2). Le nombre d'acteurs dans les warrantages AGR et intrants est plus élevé que celui observé dans le warrantage commercialisation (Tableau 2). Les commerçants et les collectivités locales sont seulement présents dans cette forme de warrantage 
Tropicultura Tropicultura 2295-8010 Volume 39 (2021) Numéro 4, 1923

(Tableau 2). L'acteur étatique est le SCDA qui joue un rôle d'appui technique et de fourniture d'intrants.

Tableau 2 : Types d'acteurs et nature des promoteurs en fonction des formes de warrantage

\begin{tabular}{|c|c|c|c|c|}
\hline \multirow{2}{*}{$\begin{array}{l}\text { Nature du promoteur } \\
\text { Types d'acteurs }\end{array}$} & & \multicolumn{2}{|l|}{ Projet } & \multirow{2}{*}{$\begin{array}{l}\text { OP } \\
\text { Warrantage } \\
\text { commercialisation }\end{array}$} \\
\hline & & $\begin{array}{l}\text { Warrantage } \\
\text { AGR }\end{array}$ & $\begin{array}{l}\text { Warrantage } \\
\text { intrants }\end{array}$ & \\
\hline Déposants & & + & + & + \\
\hline Producteurs & & + & + & + \\
\hline Collectivités locales & & + & - & - \\
\hline Fournisseurs de crédit (IMF) & & + & + & + \\
\hline Commerçants & & + & - & - \\
\hline \multirow[t]{2}{*}{$\begin{array}{l}\text { Service déconcentré de l’État } \\
\text { (SCDA) }\end{array}$} & $\begin{array}{l}\text { Fourniture } \\
\text { d'intrants }\end{array}$ & - & + & + \\
\hline & Appui technique & + & + & - \\
\hline
\end{tabular}

Légende : Impliqué : (+) ; Non impliqué : (-)

Les rôles des types d'acteurs sont définis par les conventions ou protocoles de partenariat. Ils varient suivant les types d'acteurs dans chaque forme de warrantage (tableau 3). Du tableau 3, il ressort que les rôles des types d'acteurs sont complémentaires et reflètent la synergie d'actions entre les acteurs dans chaque forme de warrantage.

Les promoteurs de warrantage ayant le statut de projet ne jouent qu'un rôle de facilitation et de renforcement des capacités des unités de gestion du processus. Par contre, le promoteur qui a le statut d'OP est le maître d'œuvre et le maître d'ouvrage du processus de warrantage. Il joue le rôle des collectivités locales, du SCDA et du comité de gestion, absents dans son dispositif. Les actions synergiques s'inscrivent donc dans une logique de rationalité définie par rapport à la coordination des activités du warrantage (Tableau 3).

Les formes actuelles de warrantage sont caractérisées par l'implication des institutions de microfinance, les organisations des producteurs et les services d'encadrement de l'État jouant respectivement le rôle de mise en place du crédit, de l'établissement de la caution solidaire et de la mise en marché et de la fourniture d'appuis-conseils en conservation des produits warrantés.

Tableau 3 : Rôles des acteurs dans la mise en œuvre du warrantage 


\begin{tabular}{|c|c|c|c|}
\hline \multirow{2}{*}{$\begin{array}{l}\text { Principaux } \\
\text { acteurs }\end{array}$} & \multicolumn{3}{|l|}{ Formes de warrantage } \\
\hline & Warrantage AGR & Warrantage intrants & Warrantage commercialisation \\
\hline Promoteur & $\begin{array}{l}\text { Facilitateur du processus } \\
\text { Construction d'infrastructures } \\
\text { de stockage } \\
\text { Renforcement de capacités } \\
\text { des comités de gestion }\end{array}$ & $\begin{array}{l}\text { Facilitateur des relations } \\
\text { multi-acteurs } \\
\text { Renforcement de capacités } \\
\text { des OP } \\
\text { Mise en place d'un fonds de } \\
\text { garantie }\end{array}$ & $\begin{array}{l}\text { Maître d'œuvre et d'ouvrage } \\
\text { Collecte, suivi et } \\
\text { commercialisation des stocks de } \\
\text { produits warrantés } \\
\text { Délivrance du certificat de dépôt } \\
\text { et de paiement } \\
\text { Sensibilisation des producteurs } \\
\text { Remboursement du crédit et } \\
\text { paiement des ristournes } \\
\text { éventuelles }\end{array}$ \\
\hline $\begin{array}{l}\text { Institution } \\
\text { de crédit } \\
\text { (IMF) }\end{array}$ & $\begin{array}{l}\text { Suivi de l'évolution des prix } \\
\text { des céréales } \\
\text { Octroi de crédit à un taux } \\
\text { d'intérêt mensuel de } 2 \% \\
\text { Appui-conseil aux producteurs } \\
\text { sur la période de déstockage } \\
\text { Recouvrement des crédits } \\
\text { octroyés dans le cadre du } \\
\text { warrantage } \\
\text { Financement des actions de } \\
\text { mobilisation et de } \\
\text { sensibilisations des OP } \\
\text { Détention d'une clé de } \\
\text { magasin } \\
\text { Prélèvement de } 200 \text { FCFA/ } \\
\text { sac pour les frais de stockage } \\
\text { et des taxes de } \\
\text { développement local (TDL) }\end{array}$ & $\begin{array}{l}\text { Octroi de crédit (3 sacs de } \\
\text { maïs pour les intrants d'un } \\
\text { ha) à un taux d'intérêt } \\
\text { mensuel de } 1,25 \% \\
\text { Détention d'une clé de } \\
\text { magasin } \\
\text { Suivi de l'évolution des prix } \\
\text { sur le marché } \\
\text { Détention du fonds de } \\
\text { garantie }\end{array}$ & $\begin{array}{l}\text { Octroi de crédit (à } 80 \% \text { au } \\
\text { moins de la valeur du stock au } \\
\text { prix du marché) à un taux } \\
\text { d'intérêt mensuel de } 2 \% \\
\text { Détention d'une clé de magasin }\end{array}$ \\
\hline $\begin{array}{l}\text { Comité de } \\
\text { gestion }\end{array}$ & $\begin{array}{l}\text { Bénéficiaires du crédit AGR } \\
\text { Libération des frais de } \\
\text { stockage et des TDL ( } 200 \\
\text { FCFA/ sac) } \\
\text { Suivi du conditionnement et } \\
\text { du stockage des produits } \\
\text { Appel de fonds et paiement } \\
\text { des crédits } \\
\text { Négociation des marchés de } \\
\text { ventes groupées des produits } \\
\text { Vente des produits après les } \\
\text { consentements des adhérents } \\
\text { Recouvrement des crédits } \\
\text { Gestion avec la mairie des } \\
\text { ressources issues de } \\
\text { l'opération }\end{array}$ & $\begin{array}{l}\text { Constitution des stocks de } \\
\text { produits warrantés } \\
\text { Délivrance du certificat de } \\
\text { dépôt } \\
\text { Maître d'ouvrage et maître } \\
\text { d'œuvre du processus } \\
\text { Commercialisation du } \\
\text { produit } \\
\text { Remboursement du crédit et } \\
\text { restitution du surplus aux } \\
\text { déposants }\end{array}$ & \\
\hline $\begin{array}{l}\text { Collectivités } \\
\text { locales }\end{array}$ & $\begin{array}{l}\text { Maître d'ouvrage du } \\
\text { mécanisme du warrantage } \\
\text { Installation d'un comité de } \\
\text { gestion par un arrêté }\end{array}$ & & \\
\hline
\end{tabular}




\begin{tabular}{|c|c|c|c|}
\hline SCDA & $\begin{array}{l}\text { Contrôle de qualité et suivi } \\
\text { des produits warrantés }\end{array}$ & $\begin{array}{l}\text { Contrôle de qualité et suivi } \\
\text { des produits warrantés } \\
\text { Fourniture d'intrants }\end{array}$ & \\
\hline Producteurs & $\begin{array}{l}\text { Déposants du produit (maïs) } \\
\text { Bénéficiaires du crédit AGR } \\
\text { Libération des frais de } \\
\text { stockage et des TDL }\end{array}$ & $\begin{array}{l}\text { Producteurs et déposants du } \\
\text { maïs } \\
\text { Bénéficiaire du crédit intrant }\end{array}$ & $\begin{array}{l}\text { Déposants du maïs } \\
\text { Bénéficiaire du crédit }\end{array}$ \\
\hline
\end{tabular}

\section{Inadéquation du warrantage face au changement climatique}

Les difficultés et risques dans la mise en œuvre du warrantage sont relatives à la non maitrise des prix sur le marché, la faible production, l'endettement des déposants, les pertes post-récoltes sur des légumineuses warrantées. Les causes de ces difficultés et risques sont diverses et certaines sont liées au changement climatique (Tableau 4). 
Adaptation des formes de financement agricole au changement climatique : cas ...

\section{Tableau 4 : Relation des risques/ difficultés du warrantage avec le changement climatique}

\begin{tabular}{ll}
\hline Difficultés/ risques & Causes selon les acteurs \\
& $\begin{array}{l}\text { Relation avec le } \\
\text { changement climatique ( } \\
\%)\end{array}$ \\
\hline
\end{tabular}

\begin{tabular}{|c|c|c|}
\hline \multirow[t]{3}{*}{$\begin{array}{l}\text { Non maîtrise des prix sur le } \\
\text { marché }\end{array}$} & $\begin{array}{l}\text { Inexistence des données sur l'évolution et la } \\
\text { prévision des prix }\end{array}$ & 3 \\
\hline & $\begin{array}{l}\text { Absence d'information sur le comportement du } \\
\text { climat qui détermine la production }\end{array}$ & 95 \\
\hline & Inorganisation des producteurs & 1 \\
\hline \multirow[t]{7}{*}{ Faible production warrantée } & Poches de sécheresse & 100 \\
\hline & Incertitude sur les périodes probables de semis & 100 \\
\hline & $\begin{array}{l}\text { Instabilité des périodes de démarrage et d'arrêt } \\
\text { des pluies }\end{array}$ & 100 \\
\hline & Non maîtrise du calendrier agricole & 100 \\
\hline & $\begin{array}{l}\text { Difficultés d’accès aux variétés tolérant la } \\
\text { sécheresse }\end{array}$ & 100 \\
\hline & Faible rendement des vivriers & 100 \\
\hline & Destruction des cultures par des inondations & 100 \\
\hline \multirow[t]{4}{*}{ Endettement des déposants } & Baisse drastique des prix sur le marché & 10 \\
\hline & $\begin{array}{l}\text { Absence d'un système d'indemnisation des } \\
\text { risques climatiques }\end{array}$ & 95 \\
\hline & $\begin{array}{l}\text { Non pratique d'AGR par certains déposants, } \\
\text { augmentant leur risque d'impayés }\end{array}$ & 20 \\
\hline & Retard dans la constitution des stocks & 30 \\
\hline \multirow[t]{3}{*}{$\begin{array}{l}\text { Pertes des légumineuses } \\
\text { warrantées (niébé, arachide) }\end{array}$} & $\begin{array}{l}\text { Inexistence de magasins sécurisés dans les } \\
\text { villages }\end{array}$ & 5 \\
\hline & Infestation des stocks par des ravageurs & 80 \\
\hline & $\begin{array}{l}\text { Difficulté d'accès aux magasins aux } \\
\text { infrastructures de stockage }\end{array}$ & 0 \\
\hline
\end{tabular}

De l'analyse de ce tableau, il ressort que sur les 17 causes de difficultés et risques, environ $60 \%$ de ces causes sont liées directement au changement climatique (Tableau 4). L'incertitude autour des 
périodes de semis, de démarrage et d'arrêt des pluies ne permettent pas aux producteurs de mieux préparer la campagne agricole et de mettre en terre les cultures à bonne date. En corollaire, les rendements sont faibles de même que les productions obtenues, limitant ainsi la capacité de dépôt des producteurs. De plus, l'incertitude climatique ne facilite pas la prévision de l'offre des produits sur le marché et ne permet pas d'anticiper les risques de mévente. Malheureusement, les stocks ne sont pas assurés par défaut d'implication des compagnies d'assurance dans le dispositif de mise en œuvre du warrantage.

\section{Discussion}

Repenser les formes de warrantage face aux enjeux du changement climatique

Les objectifs des formes de warrantage rencontrées au Bénin sont presque identiques à ceux que l'on trouve globalement en Afrique de l'Ouest et en Afrique de l'Est même si on note certaines spécificités entre les formes de warrantage $(13 ; 17 ; 18 ; 19)$. Ces auteurs ont montré que l'opération de warrantage est accompagnée de risques d'endettement des producteurs, de risques liés au marché et de risques climatiques. Ce qui corrobore les résultats de cette étude qui a révélé que les formes de warrantage au Bénin sont exposées à des risques liés au marché, des risques climatiques expliquant la faible production, etc. Dans un rapport de recherche réalisée par OXFAM au Burkina-Faso sur le warrantage paysan, il a été prouvé que les expériences de warrantage dans la région Centre Nord sont exposées aux risques climatiques et à une production céréalière déficitaire (20) ; ce qui est également ressorti de la présente étude réalisée au Bénin.

Au Bénin, l'analyse des types d'acteurs impliqués dans les formes de warrantage et de leurs rôles montre qu'aucun acteur de prévisions climatiques ni d'assurance n'est impliqué dans la mise en œuvre du warrantage. Les rôles des acteurs impliqués sont souvent limités à la caution solidaire, à la recherche de marché, au stockage et à la conservation, à la fourniture d'intrants et à la mise en place de crédit. Ainsi, l'absence, dans le dispositif, des acteurs capables de fournir des informations climatiques aux producteurs et de couvrir les risques ne permet pas à ces derniers d'anticiper les risques climatiques dans la production et la mise en marché des produits warrantés. L'absence d'une compagnie d'assurance dans le dispositif expose toujours les producteurs aux aléas du marché et aux risques climatiques. L'implication d'une compagnie d'assurance rassurerait les producteurs et constituerait une garantie pour les institutions financières. De ce fait, les formes de warrantage mises en œuvre au Bénin ne sont pas adaptées aux défis du changement climatique comme le recommandent certains auteurs (1).

Le même constat a été fait par certains auteurs qui avaient révélé l'absence des services de prévisions météorologiques dans un système d'innovation tel que le conseil à l'exploitation familiale (CEF) (5). Ces derniers précisent que ces acteurs sont incontournables pour combler les besoins actuels des producteurs face aux aléas climatiques. Certains auteurs renchérissent en disant que l'un des deux ensembles nécessaires aux conseils agricoles est constitué des prévisions météorologiques et saisonnières et des informations sur les tendances climatiques à long terme (21). Ainsi, pour mieux réviser les modèles de développement, il est important que le défi du changement climatique soit pris en compte dans les innovations (1). Ce qui n'est pas le cas dans la mise en œuvre des formes de warrantage au Bénin. Cet état de chose pourrait être dû à deux facteurs. Premièrement, les promoteurs visent prioritairement la résolution du problème du bradage des produits agricoles et du financement en occultant les questions climatiques. Deuxièmement, il existe une faible collaboration entre les acteurs de développement et les acteurs climatiques. Cette faible collaboration entre ces 
Adaptation des formes de financement agricole au changement climatique : cas ...

acteurs constitue une voie d'obstruction d'accès aux connaissances soutenant la prise de décisions adaptatives face au changement climatique (22).

Par ailleurs, les conséquences financières telles que l'endettement des déposants, la flambée des prix, les inondations, etc. qualifiées de catastrophes ne sont pas couvertes compte tenu de l'absence d'une compagnie d'assurance dans le dispositif des formes de warrantage au Bénin. Ce résultat rejoint ceux de certains auteurs qui clarifient qu'en France, les contrats d'assurance de biens garantissent la prise en charge des dommages causés par des facteurs naturels ou non naturels (23). Certains auteurs ont confirmé les résultats de cette étude en montrant qu'il existe plusieurs types de risques liés au warrantage (17). Toutefois, ces derniers notifient que certains risques tels que le risque prix et le risque rendement se compensent par une sorte d'assurance naturelle en ce sens que la baisse de rendement augmente le prix des produits warrantés. Cette assertion n'est pas toujours vérifiée car la disparité ou la mauvaise répartition des pluies due au changement climatique peut négativement affecter les rendements d'une culture d'une zone sans affecter le rendement d'autres zones. De ce fait, l'assurance pourra compenser la perte de revenu liée à la volatilité des prix sur le marché et aux aléas climatiques dans la zone concernée (17).

En somme, les formes de warrantage mises en œuvre au Bénin ne prennent pas en compte les risques climatiques. La non couverture des risques liés à l'opération justifie parfois la non adoption de l'opération de warrantage par les agriculteurs et la réticence des institutions financières à mettre le crédit en place $(14 ; 19)$. Ainsi, la prise en compte des risques liés à l'opération de warrantage est indispensable pour la pérennisation et la durabilité du système de warrantage. De ce fait, il apparaît impérieux d'impliquer de nouveaux acteurs tels que les compagnies d'assurance et les services météorologiques.

Vers une restructuration du dispositif de warrantage face aux enjeux du changement climatique

L'analyse des formes de warrantage mises en œuvre au Bénin a montré des limites pour permettre aux producteurs et productrices de faire face au changement climatique. La restructuration du dispositif devient impérative pour relever les défis du changement climatique. Elle impose l'implication de nouveaux acteurs dans les dispositifs mis en place pour conduire le warrantage (1 ; 23). Les nouveaux types d'acteurs sont les services météorologiques et les compagnies d'assurance (5; 21). L'intégration de ces acteurs imposeront de nouveaux rôles et de nouvelles relations dans le système multi-acteurs.

En ce qui concerne les services météorologiques, ils fourniront des informations sur les prévisions climatiques et météorologiques aux producteurs et productrices. Les canaux de diffusion des informations climatiques doivent être adaptés aux contextes socioéconomiques des producteurs (24). Les informations climatiques pourraient être fournies sous forme de SMS (short message services), d'email, d'audio, etc. à travers des téléphones mobiles (24). Les périodes de poches de sécheresse, les périodes de semis, la période de démarrage et d'arrêt des pluies pourront être également communiqués aux producteurs et productrices au début de la campagne. Ce qui permettra à ces derniers de développer des stratégies d'adaptation et d'ajuster les périodes des différentes opérations culturales (25).

Selon ces auteurs, les prévisions sur la fréquence et la quantité de pluie, la température, l'insolation, la direction et la vitesse du vent, etc. sont importantes pour les agriculteurs afin de réduire leur vulnérabilité face au changement climatique (25). Ils ajoutent que la fourniture des prévisions 
climatiques aux producteurs réduit le coût de production et améliore le rendement du maïs. Les services météorologiques et climatiques travailleront de concert avec les secteurs de développement agricole (SCDA) pour réviser le calendrier agricole et l'adapter aux réalités climatiques avant le démarrage de chaque campagne. Ainsi, les services météorologiques et climatiques seront mis en relation avec les SCDA.

Quant aux SCDA, en plus des rôles classiques, ils intégreront les informations ou prévisions climatiques dans leurs appuis-conseils aux OP. Ils évalueront les pertes ou dommages causés aux stocks warrantés ou aux productions agricoles du fait des risques climatiques pour les transmettre aux compagnies d'assurance. Ainsi, les SCDA pourront prédire l'évolution des prix sur le marché pour la campagne suivante compte tenu de la tendance des productions agricoles (5).

Les compagnies d'assurance couvriront les risques aussi bien liés au marché, à la récolte que climatiques qui pourraient subvenir au cours de l'opération de warrantage. Elles travailleront également avec les secteurs de développement agricole (SCDA) pour l'estimation des dommages causés par les sinistres, le marché, les récoltes et le changement climatique. La souscription à une assurance est une alternative pour faire face aux risques qui entachent l'opération et qui aggravent la situation des déposants (20). La possibilité de transférer les risques à un assureur peut se faire à travers des contrats d'assurance qui peuvent couvrir les aléas affectant la production et le revenu des déposants (17).

Toutefois, la prise en compte de l'assurance climatique dans la mise en œuvre du warrantage nécessitera initialement des investissements importants de la part de l'EÉtat ou des partenaires externes à l'opération (par exemple, les projets de développement) (18). À cet effet, les OP feront un abonnement auprès des compagnies d'assurance et recevront des informations climatiques du service météorologique. Les modalités d'abonnement des OP ou des producteurs dans les compagnies d'assurance pourraient être discutées pour réviser les conditions d'éligibilité en faveur des déposants. Au Bénin, l'Assurance Mutuelle Agricole du Bénin (AMAB) est bien adaptée pour accompagner le warrantage (26).

L'AMAB offre une assurance multirisque des récoltes en général et couvre particulièrement les risques climatiques qui affectent la production agricole (27). Toutefois, le bénéfice de ces avantages nécessite que les producteurs remplissent certaines conditions telles que la souscription à l'assurance agricole multirisque, la production du maïs, de cajou, de coton, des racines et tubercules (igname et manioc) et des légumineuses (arachide et niébé). Les formalités d'appartenance aux compagnies et le paiement de la prime d'assurance (12 euros) couvrant un semestre (27). Ces conditions deviennent de nouvelles difficultés pour les producteurs agricoles qu'il faut intégrer dans les charges de warrantage. En somme, les compagnies d'assurance prendront en charge les pertes ou dommages liés aux risques climatiques, économiques et humains pour dédommager les déposants, membres des OP. Elles rembourseront les dettes aux IMF et rétrocéderont le reste aux déposants.

Les IMF et les producteurs agricoles maintiendront leurs rôles dans le dispositif. Toutefois, ils bénéficieront des impayés auprès des compagnies d'assurance. Les producteurs pourront recevoir des informations météorologiques et climatiques.

En définitive, l'intégration du défi du changement climatique dans les innovations permettront d'aller vers une politique d'innovation climatique (1). Les enjeux du changement climatique 
apparaissent de nos jours comme des éléments perturbateurs des systèmes d'innovation tel que le warrantage $(1 ; 5)$. Ils imposent donc aux modèles de développement, la restructuration du dispositif de mise en œuvre des formes de warrantage à travers la prise en compte des services de prévisions météorologiques et climatiques et des compagnies d’assurance pour la couverture des risques (25).

Ces nouveaux acteurs travailleront avec les autres acteurs (producteurs, IMF, SCDA et comité de gestion) pour adapter les formes de financement agricole en général et les formes de warrantage en particulier. L'intégration des nouveaux acteurs dans le dispositif modifiera la structure actuelle et affectera les rôles entre les acteurs (Figure 2). Elle pourrait engendrer des coûts additionnels pour les producteurs. Toutefois, l'intégration des appuis conseils en relation avec les risques climatiques offerts par le SCDA n'engendrait pas de coût additionnel aux producteurs. Mais, elle facilitera la mise en relation des OP avec les services météorologiques. Les frais de déplacement pourraient être pris en charge par les OP. La fourniture d'informations climatiques pourrait être intégrée dans les services régaliens des services météorologiques (25). Les modalités de collaboration dans la mise en œuvre du warrantage qui inclut les défis du changement climatique doivent être discutées entre les acteurs.

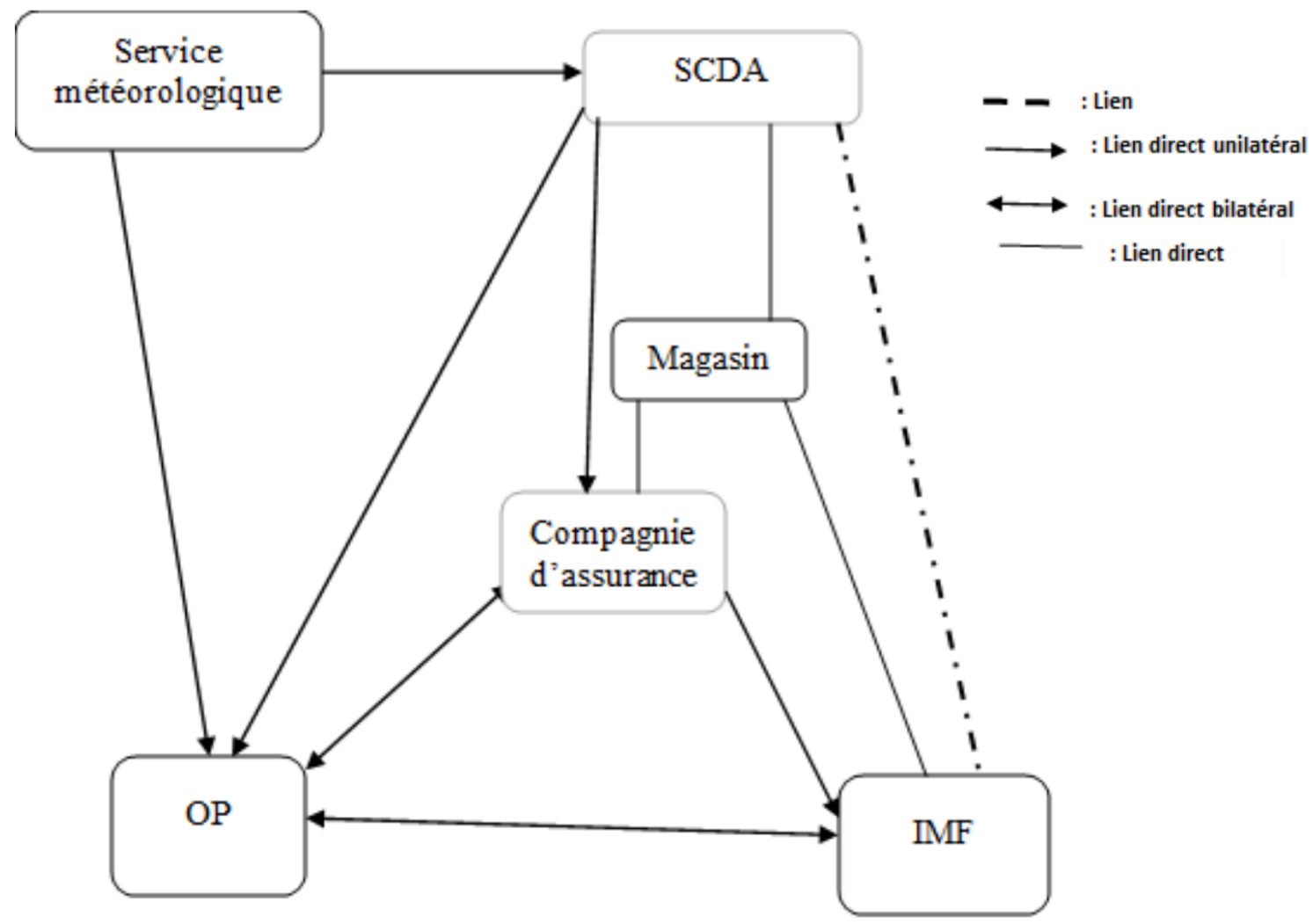

Figure 2 : Dispositif alternatif pour un warrantage climato-sensible

\section{Conclusion}

Les formes de warrantage au Bénin sont peu adaptées aux enjeux actuels du changement climatique. Certes, ces formes de warrantage ne visent pas directement la réduction des effets néfastes du changement climatique mais elles s'inscrivent dans un environnement global affecté 
par les enjeux climatiques. Dès lors, elles doivent intégrer les risques climatiques en impliquant les services météorologiques et climatiques et les compagnies d'assurance. Les premiers fourniront aux producteurs et productrices des prévisions climatiques et de production en collaboration avec le secteur de développement communal. Les secondes couvriront les risques liés à l'opération de warrantage.

En définitive, les formes de financement agricole en général et de warrantage en particulier nécessitent l'implication de nouveaux acteurs pour réduire la vulnérabilité des producteurs et productrices au changement climatique. Les politiques et les partenaires du développement agricole sont donc interpellés pour une prise de conscience climatique dans le développement des innovations.

\section{Bibliographie}

1- Touzard J-M., 2017, Innover face au changement climatique. Innovations. 3, 5-13. https://www.cairn.info/revue-innovations-2017-3-page-5.htm

2- Sossou C. H., 2015, Le financement de l'agriculture au Bénin : stratégies de gestion et d'adaptation des exploitations agricoles. (Thèse de doctorat en français). Belgique, Université de Liège-Gembloux Agro-Bio Tech. 181 p. https://orbi.uliege.be/bitstream/2268/188999/1/ SOSSOU Comlan_Herv \%C3 \%A9 Version \%20D \%C3 \%A9finitive-8-12-2015.pdf

3- Oloukoi J., Yabi I., Houssou C. S., 2019, Perceptions et stratégies paysannes d'adaptation à la variabilité pluviométrique au Centre du Bénin. Int. J. Biol. Chem. Sci. 13, 1366-1387. https://www.ajol.info/index.php/ijbcs/article/view/189565

4- FAO, FIDA, OMS, PAM, UNICEF., 2018, L'État de la sécurité alimentaire et de la nutrition dans le monde 2018. Renforcer la résilience face aux changements climatiques pour la sécurité alimentaire et la nutrition. Rome, FAO, 218p. http://www.fao.org/3/I9553FR/i9553fr.pdf

5- Baco M., Akpata M. M. S., Egah J., Affoukou K. T., Moumouni I., Nouatin G. S., 2019, Adaptation du conseil à l'exploitation familiale au changement climatique : cas du bassin cotonnier du Nord Bénin pp 269- 283. In : M. Soumaré, M. Havard, Les zones cotonnières africaines : dynamiques et durabilité. Actes du Colloque de Bamako, Novembre 2017, EDIS, Bamako, Mali, 399p. https://agritrop.cirad.fr/593138/1/Actes_Colloque_Coton_Bamako \%20Mali \%202019\%20PASE \%20II.pdf

6- O'Neill A., 2021 : Share of economic sectors in gross domestic product (GDP) in Benin 2020. https://www.statista.com/statistics/795081/share-of-economic-sectors-in-the-gdp-in-benin/

7- MAEP, 2018, Stratégie Nationale du Conseil Agricole, 92 p. https://zoomagro.com/wp-content/ uploads/2019/07/LIVRE-SNCA-2.pdf

8- Bediyé P. \& Manigui A. S., 2017, Stratégie nationale de promotion des filières agricoles intégrant l'outil clusters agricoles. Document final, 73 p.

9- Sossou K. B., Fok M., 2019, Crédit de trésorerie des producteurs de coton au centre du Bénin : modalités et conséquences. Cah. Agric. 28, 1-9. https://www.cahiersagricultures.fr/articles/cagri/ full html/2019/01/cagri190031/cagri190031.html 
Adaptation des formes de financement agricole au changement climatique : cas ...

10- Andres L. \& Lebailly P., 2013, Le financement rural du Niger, élément clé du développement. 11 p. https://orbi.uliege.be/bitstream/2268/150754/1/Ludovic_Andres_Le \%20financement \%20rural \%20au \%20Niger \%20final_ORBI.pdf

11- Bryan E., Deressa T. T., Gbetibouo G. A., Ringler C., 2009, Adaptation to climate change in Ethiopia and South Africa: options and constraints. Environmental science \& policy. 12, 413-426. https://www.sciencedirect.com/science/article/abs/pii/S1462901108001263

12- Egah J., Baco M. N., Tossou R. C., 2016, Déterminants de l'adoption des formes de warrantage du maïs au Bénin. Science et technique, Lettres, Sciences sociales et humaines. 2, 71- 86. https://bec.uac.bj/publication/4917/Article

13- Egah J., Baco M. N., Moumouni M. I., Akponikpe P. B., Yegbemey R. N., Tossou R. C., 2014, Performance of Institutional Innovation: The Case of Maize-Related Warrantage in Benin, West Africa. International Journal of Agriculture Innovations and Research, 3(2), 473- 479. https://ijair.org/administrator/components/com_jresearch/files/publications/IJAIR_936_Final.pdf

14- Egah J., Baco M. N., Yegbemey N. R., Tossou R. C., 2015, Analysis of organizational innovations adoption: cases of the different forms of maize-related warrantage in Benin. British Journal of Education, Society \& Behavioural Science. 6, 227-240. www.sciencedomain.org/download/ Nzc4N0BAcGY.pdf

15- Egah J., Baco M. N., Moumouni I. M., 2019, La biodiversité de l'igname survit-elle encore dans un contexte de changement climatique au Nord-Bénin pp 292-312. In : M. Behnassi, O. Barrière, J. Stoessel-Ritz, F. Arib, C. Prévil (Editeurs), Les systèmes socio-écologiques en Afrique du Nord et de l'Ouest face au changement global: Gouvernance, adaptation, viabilité et résilience », CERES Publishing (1ère Edition), Maroc, 399p. https://www.ceres-center.org/book-systemesecologiques?lang $=\mathrm{fr}$

16- Weikmans R., 2016, Dimensions éthiques de l'allocation du financement international de l'adaptation au changement climatique. VertigO- La revue électronique en sciences de l'environnement. 16, 1-32. https://journals.openedition.org/vertigo/17677

17- Duffau A., Lagandré D., Chetaille A., Rozenkopf I., Horréard G., Oggeri B., 2011, Gestion des risques agricoles par les petits producteurs : Focus sur l'assurance récolte indicielle et le warrantage. Document de travail 113, GRET, 86 p. https://www.gret.org/publication/gestion-desrisques-agricoles-par-les-petits-producteurs-focus-sur-lassurance-recolte-indicielle-et-lewarrantage/

18- Duffau A., Lagandré D., Chetaille A., Rozenkopf I., Horréard G., Oggeri B., 2011, Assurance indicielle et warrantage, quel intérêt pour les petits agriculteurs ? : Analyses d'expériences en Afrique de l’Est et en Inde. Coll. Études et travaux, série en ligne n 28, Éditions du Gret, 44 p. https://www.gret.org/publication/assurance-indicielle-et-warrantage-quel-interet-pour-les-petitsagriculteurs-analyses-dexperiences-en-afrique-de-lest-et-en-inde/

19- Mackiewicz-Houngue M., Renaudin M., Valleur R., Tokpa A., Amona N., 2014, Crédit intrant et crédit warrantage au Togo : Quels enseignements ? Réflexion concernant les différentes modalités de crédit proposées dans le cadre du projet d'appui au développement de la filière céréalière au Togo : atouts, contraintes et pistes d'amélioration. 32 p. https://www.avsf.org/public/posts/1849/ 
credit_filiere_cereales_togo_avsf_2015.pdf

20- Garrido E. S., Sanchez I. S., 2015, Warrantage paysan au Burkina- Faso : Accès au crédit par le biais des stocks de proximité. Rapport de recherche OXFAM, 61 p. https://www.oxfam.org/fr/ publications/warrantage-paysan-au-burkina-faso

21- Christoplos I., 2010, Climate information and agricultural advisory services: A square peg in a round hole? Rural Development News. 2, 7-13. https://www.diis.dk/en/research/climateinformation-and-agricultural-advisory-services

22- Boyer J., 2016, L'implication des acteurs de la recherche dans les processus d'adaptation au changement climatique: l'exemple des régions viticoles françaises. Innovations. 3, 147- 171. https://www.cairn.info/revue-innovations-2016-3-page-147.htm

23- Jouzel J. \& Michelot A., 2016, La justice climatique : enjeux et perspectives pour la France. Journal officiel de la république française, NOR: CESL1100010X, 114 p. http://www.lecese.fr/sites/ default/files/pdf/Avis/2016/2016_10 justice_climatique.pdf

24- Yegbemey R. N. \& Egah J., 2021, Reaching out to smallholder farmers in developing countries with climate services: A literature review of current information delivery channels. Climate Services 23, 100253. https://doi.org/10.1016/j.cliser.2021.100253

25- Yegbemey R. N., Aloukoutou A. M. and Aïhounton G. B. D., 2020, The Impact of Short Message Services (SMS) Weather Forecasts on Cost, Yield and Income in Maize Production. Africa Development / Afrique et Développement. 46, 163-188.

26- Kestemont M.-P. \& Paul E., 2020, Le rôle des mutuelles de santé et des initiatives d'économie sociale dans l'extension des mécanismes de protection sociale aux travailleurs ruraux du Bénin. Short-Term Policy Supporting Research (PSR), 186 p. https://www.louvaincooperation.org/sites/ default/files/2020-10/201.R \%C3 \%B4le \%20des \%20MUSA \%20et \%20IESS \%20dans \%201 \%27extension \%20de \%20la \%20protection \%20sociale.pdf

27- Sagna P., Dipama J. M., Vissin E. W., Diomandé B. I., Diop C., Chabi P. A. B., Yade M., 2021, Climate Change and Water Resources in West Africa: A Case Study of Ivory Coast, Benin, Burkina Faso, and Senegal. Climate Change and Water Resources in Africa: Perspectives and Solutions Towards an Imminent Water Crisis, 55-86. https://doi.org/10.1007/978-3-030-61225-2_4

PDF généré automatiquement le 2023-04-26 13:59:47

Url de l'article : https://popups.uliege.be/2295-8010/index.php?id=1923 